\begin{tabular}{|c|l|}
\hline Title & Chaotic itinerancy and its roles in cognitive neurodynamics \\
\hline Author(s) & Tsuda, Ichiro \\
\hline Citation & $\begin{array}{c}\text { Current opinion in neurobiology, 31, 67-71 } \\
\text { https://doi.org/10.1016/.conb.2014.08.011 }\end{array}$ \\
\hline Issue Date & 2015-04 \\
\hline Doc URL & http://hdl.handle.net/2115/61041 \\
\hline Rights(URL) & http://creativecommons.org/icenses/fy-nc-nd/4.0/ \\
\hline Type & article (author version) \\
\hline Additional Information & There are other files related to thisitem in HUSCAP. Check the above URL. \\
\hline (For 2nd revised submission)chaotic itinerancy and its $\quad$ role.pdf (Text) \\
\hline
\end{tabular}

Instructions for use 
Manuscript Number CONEUR-D-14-00079

\section{Chaotic Itinerancy and its Roles in Cognitive Neurodynamics}

Ichiro Tsuda

Research Institute for Electronic Science,

Hokkaido University

Kita-12, Nishi-7, Kita-ku, Sapporo, Hokkaido 060-0012, Japan

e-mail address: tsuda@math.sci.hokudai.ac.jp

HP: http://cls.es.hokudai.ac.jp/ tsuda/en/index.html

Tel \& fax: +81-11-706-3824

\section{Abstract}

Chaotic itinerancy is an autonomously excited trajectory through high-dimensional state space of cortical neural activity that causes the appearance of a temporal sequence of quasi-attractors. A quasi-attractor is a local region of weakly convergent flows that represent ordered activity, yet connected to divergent flows representing disordered, chaotic activity between the regions. In a cognitive neurodynamic aspect, quasi-attractors represent perceptions, thoughts and memories, chaotic trajectories between them with intelligent searches, such as history-dependent trial-and-error via exploration, and itinerancy with history-dependent sequences in thinking, speaking and writing. 


\section{Introduction}

Motivated by studies for the elucidation of the dynamical mechanism of the complex transitions observed in brain activity, many researchers have proposed conceptual frameworks for understanding such a mechanism. Among others, we proposed a neural chaotic itinerancy $[1,2,3,4,5]$, where typical cortical transitions are not merely random but are transitory and chaotic dynamics (see, for example, [6]). Chaotic itinerancy is a prerequisite for the persistence of memories during learning. In a neural network model, with recurrent connections of excitatory units under the presence of inhibitory units, the appearance of chaotic itinerancy among quasi-attractors as memories allows the network to learn new input patterns while maintaining memories. The significance of chaotic itinerancy becomes clearer in the situation of human communication. Indeed, to communicate, each person needs rapidly to construct and reconstruct history-dependent memory structures, which must not be disturbed by others' actions. One can construct coupled-neural networks to account for this situation [7], and extend them further to more realistic and biologically oriented neural networks consisting of a neuron model by Pinsky and Rinzel [8] for excitatory neurons, and another one by Wang and Buzsáki [9] for inhibitory neurons [10].

\section{Hierarchical structure of memory}

Memory is constructed in a hierarchical manner. The first step, a stage of simple memory, according to Marr [11], is realized via embedding of input patterns as attractors by means of, for example, a Hebbian learning algorithm. Auto-associative memory models are typical for the neural realization of such algorithms (see, for example, [12]). The realization of a critical state of the attractors through multiple-metastable states can produce chaotic itinerancy. Hebbian learning then strengthens the paths connecting such quasi-attractors rather than any one attractor, because of the development of dynamical trajectories via chaotic itinerancy. This is the second stage of memory, memory of the association process; namely episodic memory. The third stage was studied recently by Kurikawa and Kaneko [13], and suggests that the input-output relation is embedded as memory, thus describing the stage of relational memory between sensation and action. Such memory is dynamically represented by bifurcations of dynamical states until action is output when information of sensation is input; chaotic itinerancy appears in this bifurcating process. This stage of memory formation can be considered to be a precursor of higher-order memory formed through communication, because 
output-driven information processing is more effective than input-driven processing when people are communicating to produce reafference via preafference (see, for example, [14]).

\section{Communicating brains}

Chaotic itinerancy also appears in interacting systems, typically appearing in the brain activity of communicating people in the form of chaotic transition between synchronization and desynchronization (see, for example, [15,16,17]). Furthermore, an atmosphere generated by cooperative actions forms the basis for the creation of meaning when people are communicating. In relation to this situation, recent studies with mathematical models of neural networks that are based on a variational principle providing a constraint to the system showed the generation of functional units that is, functional differentiation with the help of chaotic itinerancy $[18,19]$.

The appearance of chaotic itinerancy in this communication paradigm is realized via the generation of metastable states [20,21] or via critical states (see, for example, [16]). Metastable states have also been proposed as the transient states of ongoing coordination dynamics in neural systems, which are linked to intermittency, chaotic itinerancy and self-organized criticality in dynamical systems [20]. In a similar way to acquisition of the robustness of critical states via synaptic learning [16], metastable states can appear in a robust manner via interacting brains in communication paradigm [21]. Thus, the appearance of metastable states or critical states is a necessary condition for chaotic itinerancy but not a sufficient condition (see [22] for mathematical conditions for the production of chaotic itinerancy). Let us see the dynamical development in a neighborhood of such states.

\section{Neutral stability and criticality}

A quasi-attractor in chaotic itinerancy is an attractor because it possesses a positive measure of attracting regions. Thus, a quasi-attractor is different from a saddle in cases without symmetry, where symmetry restricts dynamical trajectories to some subspace of a whole state space, providing the reduction of space dimension. Nevertheless, dynamical trajectories leave a quasi-attractor and become chaotic until they reach another quasi-attractor (Fig. 1). In this respect, a quasi-attractor is a Milnor attractor [23] but not a conventional attractor. However, the dynamical trajectories are trapped in a basin of attraction when a quasi-attractor as a Milnor 
attractor exists; thus, no chaotic wandering appears unless a basin is riddled. Therefore, to obtain chaotic transitions, a quasi-attractor should not be stabilized, at least, in the sense of linear stability. Then, in a neighborhood of a quasi-attractor, the dynamics begin from at least the second order, because of the linear term vanishing; $d x / d t=b x^{2}+O\left(x^{3}\right)$, where $x$ denotes a state variable and $b$ denotes a parameter. In relation to this structure of neutral stability, Kozma et al. showed, with a hierarchical model of neuropercolation, that higher moments than the second order in critical states act in giving the indices for the appearance of perception and cognition [16]. Compared with the exponential convergence in time of dynamical trajectories to a conventional attractor, caused by the presence of a linear term, the convergence to a quasi-attractor is much slower in an algebraic manner. This type of slow convergence is a realization of neutral stable states. Usually, this critical state is yielded via bifurcations, so that the dynamical system is structurally unstable and hence not observed generically. However, such a critical state can be generically observed in network systems with scale-free properties because of a mechanism similar to self-organized criticality [16,24,25]. Actually, the neuropercolation model describes robust and stable criticality in large assemblies of neural cells [16]. In such a model, intermittent transitions between synchronization and desynchronization stably occur in a critical regime [16], which is quite similar to chaotic itinerancy between synchronization and desynchronization in gap junction-coupled neural networks [17].

-- Fig. $1^{--}$

The dynamic process and its transition rule were studied in a recurrent network with excitatory neurons, each receiving inhibitory signals, a structure found in common in both the neocortex and the hippocampus, and a circle map with criticality as a transition rule was found. The criticality was robust for changing network parameters [1,2]. Here, criticality appeared in two ways: a circle map was in a critical stage between a chaotic map and a stable one, and fixed points representing memories were Milnor attractors but not conventional ones. In this critical situation, dynamical trajectories slowly approached quasi-attractors, but these can develop along divergent flows before reaching quasi-attractors. Then we call a neighborhood of a quasi-attractor an attractor ruin.

\section{Learning via critical states}


Furthermore, one can study the criticality of dynamics in neural learning in a framework of information geometry [26]. Let us consider a set of multilayer perceptrons. Learning occurs by changing the connection strengths of neuronal units. Here, a set of modifiable connections forms a variable base of coordinates, which describes the dynamics associated with learning. In neural networks, there exist symmetries such as, say, $w_{1 j}=0$ for all $j$ in a certain layer. These symmetries bring about identification of the networks. However, on such a subspace, non-identifiable networks remain, and these bring about singularities in the space of distribution of the networks. Dynamical trajectories in some subset of such a space converge to such singularities, implying a failure of learning, but in some other subset of space, divergent flows appear in a neighborhood of such singularities; this implies the presence of an appropriate learning algorithm. Thus, singularities are critical, or neutral, and appear as Milnor attractors [27]. Here, the appearance of a Milnor attractor implies the plateau phenomenon, whereby acquisition of adequate learning takes a long time.

Thus, the general framework of learning suggests that the appearance of neutral stability in the cortical neural system also plays an important role in learning of the relationship between a real-world object (signifié in French; signified in English) and its symbolic representation (signifiant in French; signifier in English). An understanding of such a relationship is essential for human intelligence capable of acquiring sufficient skills to change both a situation in the world and its meaning. In order to classify illocutionary acts, Searle [28] introduced the notion of the direction of fit', thereby determining which of the symbols (words) and objects (the world) can be the basis of a viewpoint as "origin of coordinates" or "fixed point". He applied this aspect to the classification of two categories of sentences: constative and performative sentences. Constative sentences describe situations happening in the world, for example, "there are two apples in this room", whereas performative sentences do not describe the world but change a situation in the world, for example, "put an apple on the table". In the former case, the situation in the world is the basis of the viewpoint, and therefore the direction of fit is 'words-to-the-world'; that is, from signifier to signified. On the other hand, in the latter case, the sentence changing the situation is the basis of the viewpoint, and therefore the direction of fit is 'the-world-to-words'; that is, from signified to signifier.

Searle further pointed out that one exceptional case-declarative sentences- 
remains unclassified into these two categories. Declarative sentences, for example, "we now open the workshop" contain both constative and performative sentence features. This is because declarative sentences describe the situation that has just been declared and also change the situation by the declaration. Thus, a declarative sentence possesses two bases with opposing directions and thus acts as if it is a zero vector, appearing in the case of neutral stability or criticality, as mentioned above.

Based on this theoretical minimum, it is important to study the difference in abilities between humans and animals for a deep understanding of human intelligence from the biological evolution viewpoint (see, for example, [29,30]). Matsuzawa and his colleagues investigated the language acquisition of chimpanzees with a genius chimpanzee named $\mathrm{Ai}$, in the 'Ai project' (see, for example, [31]). Ai could learn associations from colors to corresponding symbols, but she could not apply an inverse process of association from learned symbols to colors. Therefore, what Ai learned was the direction of fit, from signifier to signified, but not an opposite direction of fit. In other words, Ai acquired association, which possesses the same direction of fit as constative sentences, but could not change the world by using learned symbols. Interestingly, human infants can apply both directions of fit by learning only a single direction of fit. One hypothesis, proposed by Osawa [32], suggests that human infants learn the relationship between signifier and signified by language acquisition in declarative circumstances. If that is correct, it would be reasonable to think that learning via a neutral state or a critical state could differentiate human communication from animal communication.

In mathematical models of neural networks, a pair association of $(A, B)$ can easily be realized by means of a hetero association learning algorithm in a hetero association network. Then, for the input $A$, the output is $B$, and vice versa, and thus it is symmetric. Furthermore, if the output is expected to be $B$ for the input $A$, supervised learning could be adopted. Thus, it is well known that a certain neural network learns " $A$ implies $B$ '. However, such a model cannot produce " $B$ implies $A$ " simply by learning " $A$ implies $B$ ', as in the case of Ai's learning of symbolic representations of colors. As far as we know, a neural network model for realizing symmetric association by learning only one direction of association has not explicitly been proposed. On the other hand, a large-scale neural network generically produces chaotic transitions among learned states, i.e. chaotic itinerancy. 
Transition is symmetric in the sense of possibility, if it goes through neutral stability. In other words, the transition paths not only from $A$ to $B$ but also from $B$ to $A$ are strengthened by Hebbian learning. Therefore, the question of how the internal structure of a neural network is modified to produce chaotic itinerancy in supervised learning, such as a learning of “ $A$ implies $B$ ', must be an important topic to study in cognitive neurodynamics.

\section{Conclusion}

We emphasized the neurobiological significance of chaotic itinerancy with some conceptual frameworks. Chaotic itinerancy was proposed to play essential roles in the hierarchical formation of memory and also in learning the meaning of symbols.

\section{Acknowledgments}

This work was partially supported by a Grant-in-Aid for Scientific Research on Innovative Areas "The study on the neural dynamics for understanding communication in terms of complex hetero systems (No.4103)" (21120002) of The Ministry of Education, Culture, Sports, Science, and Technology, Japan, and was partially supported by HFSPO (HFSP.RGP0039).

\section{References}

[1] Tsuda I: Chaotic itinerancy as a dynamical basis of hermeneutics of brain and mind. World Futures 1991, 32:167-185.

[2] Tsuda I: Dynamic link of memories-chaotic memory map in nonequilibrium neural networks. Neural Netw 1992, 5:313-326.

[3] Tsuda I: Toward an interpretation of dynamic neural activity in terms of chaotic dynamical systems. Behav Brain Sci 2001, 24:793-810, discussion 810-847.

**[4] Tsuda I: Chaotic itinerancy. Scholarpedia 2013, 8(1):4459.

This is a recent review of chaotic itinerancy for the readers in both neuroscience and dynamical systems. Similar concepts representing the transition phenomena in neural systems, which have been proposed so far, are described in comparison with chaotic itinerancy. 
[5] Kaneko K, Tsuda I: Chaotic Itinerancy. Chaos: Focus Issue on Chaotic Itinerancy 2003, 13(3): 926-936.

[6] Skarda CA, Freeman WJ: How brains make chaos in order to make sense of the world. Behav and Brain Sci 1987, 10:161-195.

[7] Li Y., Tsuda I: Novelty-induced memory transmission between two nonequilibrium neural networks. Cog Neurodyn 2013, 7(3):225-236.

[8] Pinsky PF, Rinzel J: Intrinsic and network rhythmogenesis in a reduced Traub model for CA3 neurons. J Comp Neurosci 1994, 1:39-60.

[9] Wang XJ, Buzsáki G: Gamma oscillation by synaptic inhibition in a hippocampal interneuronal network model. J Neurosci 1996, 16:6402-6413.

[10] Tsukada H, Yamaguti Y, Tsuda I: Transitory memory retrieval in a biologically plausible neural network model. Cog Neurodyn 2013, 7(5):409-416.

[11] Marr, D: Simple memory: A theory for archicortex. Phil Trans Royal Soci London 1971, 262:23-81.

[12] Amari S: Dreaming of mathematical neuroscience for a half century. Neural Netw 2013, 37:48-51.

[13] Kurikawa T, Kaneko K: Embedding responses in spontaneous neural activity shaped through sequential learning. PLOS Comp Biol 2013, 9:e1002943.

**[14] Freeman WJ: How Brains Make Up Their Minds. A Phoenix Paperback; 1999.

Freeman treats biological dynamics of self, consciousness, intention, emotion, and selection and freedom, based on his finding of chaotic transitory behaviors in various hierarchical levels of neural systems. With his ideas, one knows functional significance of chaotic activity in the brain, particularly the dynamic implications of attractor landscape and chaotic itinerancy. This book was translated in Japanese and published from Sangyo-tosho publishing company, Tokyo, in 2010. 
[15] Kawasaki M, Yamada Y, Ushiku Y, Miyauchi E, Yamaguchi Y: Inter-brain synchronization during coordination of speech rhythm in human-to-human social interaction. Scientific Reports 2013, 3:1692.

*[16] Kozma R, Puljic M, Freeman WJ: Thermodynamic model of criticality in the cortex based on EEG/ECOG data. Criticality in Neural Systems. Edited by Plenz, D, Niebur, E. Bethesda: John Wiley \& Sons; 2012:1-22.

This paper treats the hierarchies of dynamic behaviors in neural systems by means of neuropercolation models, which are proposed as appropriate tools to compute complex dynamic behaviors including chaotic transitions between synchronization and desynchronization at critical regime in effective spatiotemporal dimensions.

[17] Tsuda I, Fujii H, Tadokoro S, Yasuoka T, Yamaguti Y: Chaotic Itinerancy as a Mechanism of Irregular Changes between Synchronization and Desynchronization in a Neural Network. J. of Integra Neurosci 2004, 3:159-182.

[18] Tsuda I, Yamaguti Y, Watanabe H: Modeling the genesis of components of networks of interacting units. To be published In Advances in Cognitive Neurodynamics (IV). Springer-Verlag 2014: Proceedings of the 4th International Conference on Cognitive Neurodynamics, Sweden, June 23-27, 2014.

[19] Yamaguti Y, Tsuda I: Mathematical modeling for evolution of heterogeneous modules in the brain. To be published in Neural Netw 2014.

[20] Kelso JAS, Dumas G, Tognoli E: Outline of a general theory of behavior and brain coordination. Neural Netw 2013, 37:120-131.

*[21] Tognoli E, Kelso JAS: The metastable brain. Neuron 2014, 81:35-48.

This paper treats metastable dynamics as a prerequisite for the dynamic coordination, by means of coupled nonlinear oscillators. The authors describe how meaning is created, associated with the emergence of metastable states in coordination dynamics. The analyses are also extended to coordination dynamics in communicating people.

[22] Tsuda I: Hypotheses on the functional roles of chaotic transitory dynamics. 
Chaos 2009, 19:015113-1-015113-10.

[23] Milnor J: On the concept of attractor. Comm Math Phys 1985, 99:177-195.

[24] Levina A, Herrmann JM, Geisel T: Dynamical synapses causing self-organized criticality in neural networks. Nature Phys 2007, 3:857-860.

[25] Bak P, Tang C, Wiesenfeld K: Self-organized criticality: An explanation of the 1/f noise. Phys Rev Lett 1987, 59:381-384.

[26] Amari S, Nagaoka H: Methods of Information Geometry. AMS and Oxford University Press; 2000.

[27] Amari S, Ozeki T, Cousseau F, and Wei H: Dynamics of learning in hierarchical models - Singularity and Milnor attractor. In Advances in Cognitive Neurodynamics (II). Springer-Verlag; 2011:3-9.

[28] Searle J: Expression and Meaning. Cambridge University Press; 1979.

[29] Hatakeyama M, Tsuda I: Internal logic viewed from observation space: Theory and a case study. BioSystems 2007, 90:273-286.

[30] Pan X, Sawa K, Tsuda I, Tsukada M, Sakagami M: Reward prediction based on stimulus categorization in primate lateral prefrontal cortex. Nature Neurosci 2008, 11:703-712.

[31] Matsuzawa T: Evolution of the brain and social behavior in chimpanzees. Curr Opin Neur 2013, 23:443-449.

[32] Osawa M: Origin of sociality. Hon 2014, June issue:54-61 (in Japanese). Kodansha Ltd. 


\section{Figure Caption}

Figure 1 Schematic drawing of quasi-attractors and dynamical trajectories in chaotic itinerancy

The dynamics develops on a smooth m-dimensional manifold $M$ or an m-dimensional Euclidean space $R^{m}$, where $m \geq 4$. Different types of quasi-attractors are denoted by symbols, $A, B, C, D$, and $E$ as one possible realization of ordered motion. Each quasi-attractor possesses the inlet and the outlet chaotic trajectories, which connect such a quasi-attractor with other quasi-attractors, thereby realizing a critical state of such a quasi-attractor. Here, a narrow bundle of trajectories such as $L 1$ indicates a low-dimensional chaotic trajectory, and a broad bundle such as L2 indicates a high-dimensional chaotic trajectory. Arrows show the directions of dynamical development of trajectories. Transitions between quasi-attractors are chaotic and history-dependent. In particular, when quasi-attractors represent various synchronization states, chaotic itinerancy describes intermittent transitions between synchronization and desynchronization. 